\title{
The Case of China
}

\section{The Societal Impact of the "Whole of Government Approach"}

\author{
Wang Jing and Wang Xue
}

\begin{abstract}
This article describes, from a sociopolitical, socioeconomic, and sociocultural perspective, the governance practices of the COVID-19 epidemic control response in China. We describe that, in line with the "whole of government approach," strong resource mobilization and control of government departments, companies, and citizen communities has worked efficiently to rapidly contain the epidemic. Community participation at the grassroots level has played a decisive part. We assume that the deeply rooted collectivistic Chinese culture has made residents trust the government's decisions and comply with the prevention and control strategies. We pose some intriguing questions for more analytical comparative research. They concern the normative interpretation of the influences of sociopolitical, economic, and cultural forces, as well as the balance between "collectivism" and "individualism" in societies.
\end{abstract}

Keywords: collectivism, crisis, governance, individualism, social quality, whole of government approach

In 2020, the COVID-19 pandemic, being the largest global pandemic since 1918, spread across the world. After more than 100 years of technological evolution, vaccines and antiviral drugs have completely eliminated some infectious diseases for human beings. However, after the outbreak of COVID-19, both high-income and low-income countries still could not find a way out when facing the rapid spread of COVID-19. Due to the hidden, extremely contagious nature and high pathogenicity and lethality of the COVID-19 infection, its threat exceeds that of any natural disaster or armed conflict. After the outbreak of the pandemic, different countries have shown different approaches to emergency response. Singapore and South Korea adopted a unified organization approach from the beginning to mobilize public and private laboratories, and comprehensively and rapidly detect and track suspected cases. These actions had a positive effect when it came to curbing the transmission of COVID-19 (Abdou 2021). The United States, in line with its political-administrative structure, adopted the initially less successful state-wide, decentralized, anti-epidemic organization approach (Kettl 2020). As we will see, China adopted a top-down centralized 
governance approach to deal with the public health crisis. As part of China's pandemic governance, people largely complied with the measures taken by the Chinese government (e.g., the use of digital applications, health code measures).

In this article, we present a detailed description of the specific government approaches that were deployed in China to cope with the COVID-19 pandemic. In our description, we will make use of components of social quality theory (SQT) and the social quality approach (SQA) to describe the deployed Chinese interventions, societal agencies, and private companies, as well as and the state's relationship with the public. We analyze the societal impact of this situation in line with the dimensions deployed in the SQA: the sociopolitical and legal, the socioeconomic and financial, the sociocultural and welfare, and the socioenvironmental and ecological dimensions (IASQ 2019: 58). Our description of the complexities of the governance practices can be tracked along processes in and between these dimensions (IASQ 2019). In this article, we focus on the societal processes with respect to the first three social quality dimensions.

We start by picturing the epidemiological context: the onset, development, and course of the COVID-19 pandemic in China. We do so by presenting figures on mortality rates, case fatality rates, and vaccination coverage. Then we explain our research questions and the social quality framework on which the applied descriptive methodology is based. In the following sections, we describe our findings, which are presented in line with the three social quality dimensions. The sociopolitical and legal dimension describes the political-administrative structure, and the interventions led by the Chinese government. The sociocultural and welfare dimension describes the way in which Chinese residents were involved in the prevention and control strategies. This includes the development of community grids, societal based organizations, and volunteer groups. These reflect the organizational approaches through which individuals took part in the pandemic prevention and control strategies. In the socioeconomic and financial dimension, we picture the economic interventions of the Chinese government, as well as the compliance of companies and technology organizations participating in the pandemic control measures.

We conclude our descriptions by identifying the specific idiosyncrasy of China's comprehensive governance of the COVID-19 public health crisis. The agencies in Chinese society regarded "guaranteeing individual security, promoting general collective security and realizing common development" as their common goal (Xinhua News Agency 2020a). To control the rapid spread of the COVID-19 pandemic, they collaborated with each other and jointly made contributions to the required societal transformations. Furthermore, we emphasize, that without the extensive participation of the public and changes in individuals' behaviors, China would not have achieved today's results of pandemic prevention and control.

In times of crisis, structural (often covered) societal dynamics underpinning the quality of "the social" become uncovered and come within reach of scientific endeavors. In this article, we do not analyze and explain those underlying dynamics. We consciously limit ourselves to the description of the form, content, and synergy of 
the applied processes of governance for "the case of China." From January 2020 to September 2021, the Chinese government largely contained the spread of COVID-19 through centralized resource mobilization and reallocation. Previous studies have discussed China's performance in pandemic prevention and control from multiple angles, including from the perspective of the state's public health system and general welfare policies (Bouey 2020; Soon et al. 2021). The specific political-administrative structure of Chinese society, with its powerful centralized governance dynamics, offers specific opportunities to respond early, systematically, and efficiently to crises like the COVID pandemic. The detailed description of the events that took place in China to combat the epidemic outbreak can therefore be of interest to other countries for the purposes of future crisis management. Given the speed and efficiency of the Chinese approach to containing the pandemic, interesting questions arise regarding the specific nature and dynamics of Chinese society that have made such a response possible. These questions may deliver important points of departure for an in-depth comparative analysis of how precisely "social quality" is achieved vis-à-vis the "self-realization of individual people and the formation of collectivities" (Westbroek et al. 2020). We therefore finalize our conclusions by putting forward some challenging fundamental questions regarding this issue.

\section{Epidemiological Context}

The COVID-19 pandemic has brought unprecedented burdens on global health, and it has negatively impacted the world's economies and cultures. By 17 September 2021, the number of people infected with the COVID-19 climbed to 226 million worldwide and the number of deaths reached 4.66 million (WHO 2021). The COVID-19 pandemic in China has gone through two stages: January-March 2020 was the outbreak stage; due to the lack of initial preparation, the number of infected people surged, reaching its peak in early March (over 80,000). In late March 2020, China entered the second stage, one of prevention and control, during which local governments adopted many "unconventional" measures, including mass testing and physical distancing, which effectively controlled the spread of the disease. The infection rate continued to fall, and large-scale COVID-19 transmission was gradually controlled: the infection curve flattened gradually. From March 2020 to the present, no large-scale outbreak has been seen in China. As of 17 September 2021, there have been a total of 5,688 deaths caused by COVID-19, with the absolute death count reaching a peak of 0.13 deaths per million in mid-April 2020 and declining steadily thereafter. The government verified the death toll in April and counted previous underreporting and misreporting. The sudden increase appeared due to this statistical delay. The rate of confirmed fatalities has been maintained at 5 percent.

After December 2020, when local vaccine development and production had been realized, China's prevention and control strategy shifted from traditional home 
Daily new confirmed COVID-19 deaths per million people Shown is the rolling 7-day average. Limited testing and challenges in the attribution of the cause of death means

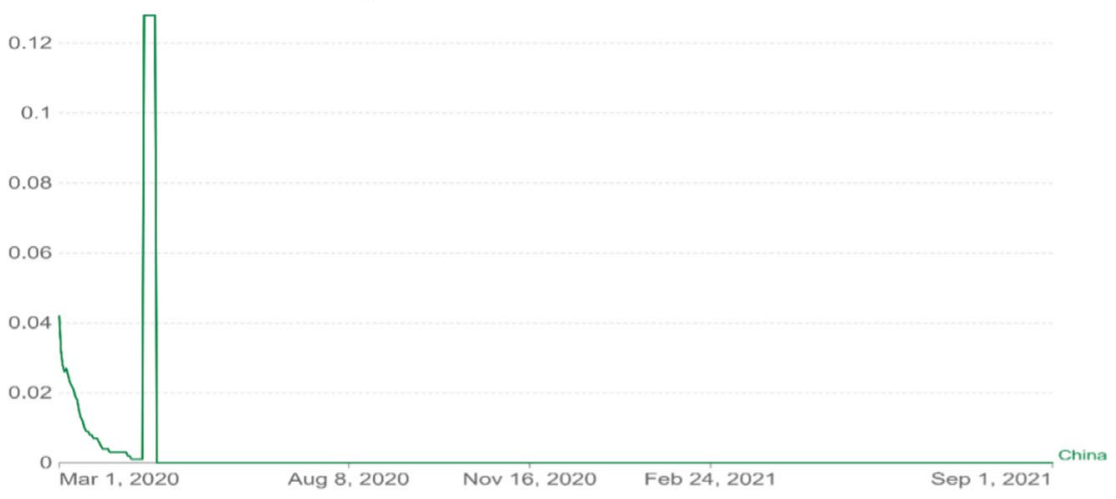

Source: Johns Hopkins University CSSE COVID-19 Data

Figure 1. Daily New Confirmed COVID-19 Deaths per Million People: 1 March 2020 to 1 September 2021 (Source: https://ourworldindata.org/coronavirus)

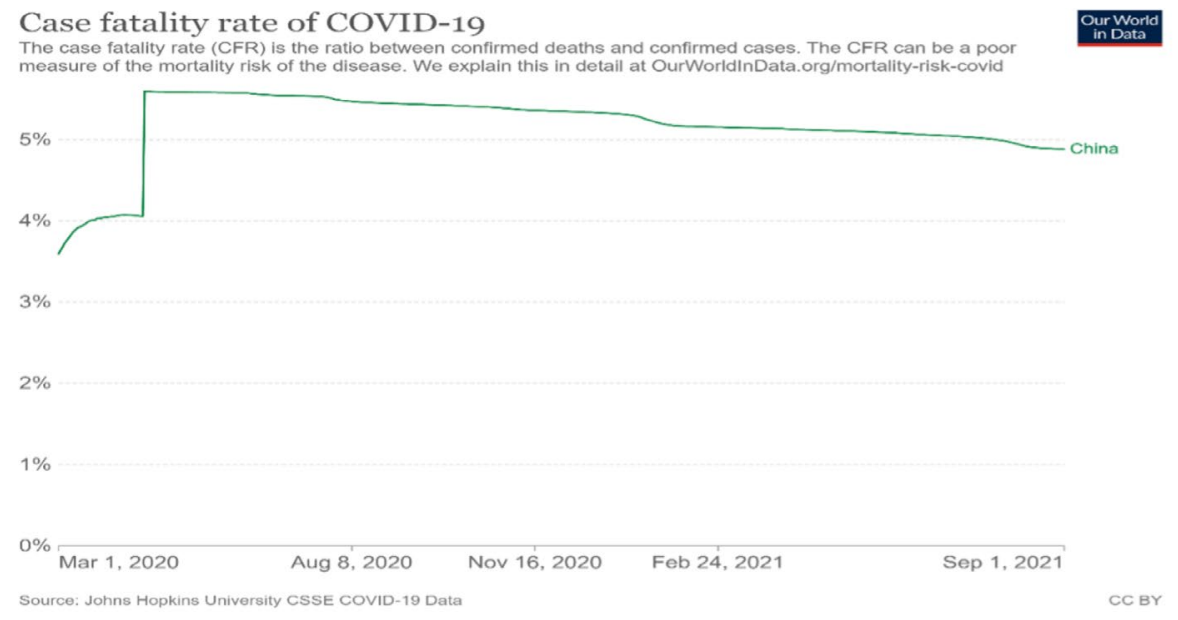

Figure 2. COVID-19 Case Fatality Rate: 1 March 2020 to 1 September 2021 (Source: https://ourworldindata.org/coronavirus)

quarantine and non-pharmaceutical interventions to mass vaccination. The Government, in an effort to expand vaccination coverage, provided fiscal and health insurance support to ensure free vaccination access to all populations. In early December, due to limited vaccine production, vaccination in batches was adopted. The first batch of vaccine recipients mainly included persons in industries and on positions with a higher risk of exposure to COVID-19 virus. The second batch of vaccine recipients are those on key positions. Since February 2021, vaccines were open to general public. By the 


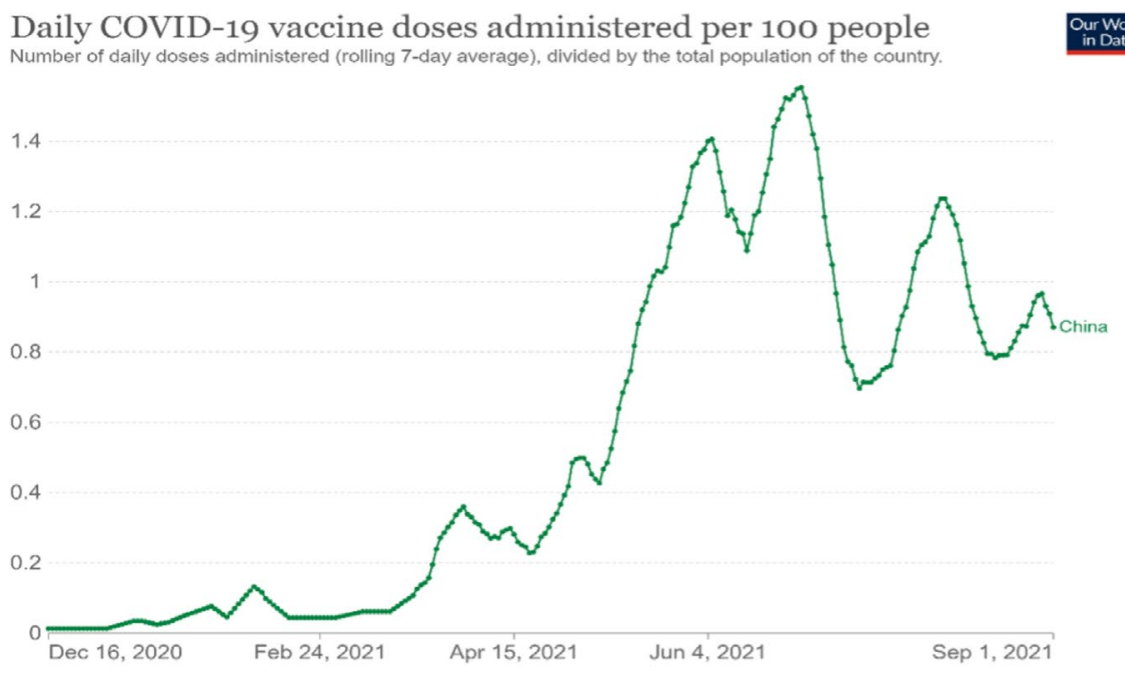

Source: Official data collated by Our World in Data. For vaccines that require multiple doses, each individual dose is counted.

Figure 3. Daily COVID-19 Vaccine Doses Administered per 100 People: 16 December 2020 to 1 September 2021 (Source: https://ourworldindata.org/coronavirus)

end of September 17, the vaccine doses administered was over 2.17 billion, which will continue to grow until herd immunity will be achieved.

In conclusion, it may be stated that China, after a dramatic beginning, has achieved rapid results in combatting the COVID-19 pandemic. In the following sections, we will describe in detail what government strategies and approaches were deployed, what measures were taken, and how people and societal organizations and companies responded to the crisis.

\section{Research Question and Methodology}

Our research question includes diverse aspects of the Chinese approaches: what is the (hierarchical) political-administrative structure of the Chinese Government; what governance approaches were deployed; what special organizations, policies and measures were set up; how was general public involved in the interventions; and how were other societal agencies and commercial companies involved.

In order to organize our descriptions, we will make use of the social quality framework. Social quality theory (SQT), born at the end of the twentieth century, is based on the ontological notion that "humans and collective entities do evolve in dialectic processes between individuals and these entities or formations, resulting in societalbased relations and structures" (Beck et al. 2012: 47). SQT is built on the ontology that the human essence is "sociality." This implies an understanding of "the social" as 
its most crucial aspect (Van der Maesen and Walker 2012). Individuals are embedded in societal relations and structures. In a dialectical sense, "the individual" and "the collective" are poles relative to each other, and they therefore constitute each other (Zhao 2011). By deploying the SQA, it becomes possible to picture the degree of "the quality of the social" in a certain place at a certain time (IASQ 2019). In other words, it becomes possible to learn "to what extent people are able to participate in societal based relationships under conditions which enhance their well-being, capabilities and potentials" (Van der Maesen and Walker 2012: 68; see also IASQ 2019). These basic notions about the interrelationships of individuals and collectivities underlie the questions we pose in our conclusions. In our descriptions of the interrelationships between the public and societal organizations (e.g., the government), we use, among others, the conditional factors of SQT: socioeconomic security, social cohesion, social inclusion, and social empowerment.

The COVID-19 pandemic is a crisis of the utmost complexity. It affects many aspects and components of society. To achieve rapid and efficient results, then, the Chinese tried to involve all related components of society, aiming to organize comprehensive, interdisciplinary, and cohesive interventions. According to the SQA, societal processes are to be understood from the perspective of four distinct dimensions: the socioeconomic and financial, the sociopolitical and legal, the sociocultural and welfare, and the socioenvironmental and ecological (IASQ 2019). Combining these dimensions in our descriptions opens the way for a multidimensional comprehensive understanding of transformation processes (Novakova 2017; Ricceri 2019). Since we do not have the data available to analyze processes in the socioenvironmental and ecological dimension, we will limit our description to the perspectives of the first three dimensions.

It should be clear that this article does not aim to achieve a better and more indepth understanding and evaluation of China's societal dynamics, which would lead to insights into the "quality of the social." To achieve this, further analytical studies would be required.

In our extensive and detailed descriptions of the societal processes that took place in China to combat the pandemic, we have made use of observations from a multitude of data resources, including laws, policy documents, reports, and scientific documents. Many observations came from the same sources. While we have included specific references for most of our observations, we have done so for all of them, given considerations of space.

\section{The Sociopolitical and Legal Dimension: The Whole of Government Approach}

The relationship between the central and local governments is a key factor in understanding crisis governance in China. There is already a vast literature on the charac- 
teristics of central-local authority relations in China, such as central-local financial relations, official appointments, and administrative controls. Some scholars deem that the relationship between the central government and local governments is of a quasifederal nature, and that local government behavior is characterized by decentralization (Qian and Weingast 2017). Especially after the introduction of the tax-sharing system in the 1980s, local governments have had full autonomy in local economic development and have had surplus distribution rights with regard to local tax revenues, something that has encouraged local governments to fully develop their respective local economies. This control, then, according to these scholars has been a key factor in China's rapid economic takeoff in the past decades (Zhou 2018). Other scholars hold the opposite view, arguing that the central government's governance capacity has not been weakened by the economic deconcentration, that the central government still holds a great deal of power in the appointment of cadres at all levels, and that the vertical accountability mechanism centered on power over personnel has given the central government a great deal of infrastructural power, shaping the behavior patterns of local governments (He et al. 2020). The latter view has been repeatedly endorsed by the governance of a number of special crises, such as the SARS epidemic in 2003 and the Wenchuan Earthquake in 2008, where the central government reduced the damage caused by the crises in the shortest possible time by the unified deployment and allocation of resources at all levels, which showcased the strength of China's political system (Zhong 2020; Zhong and Lin 2018).

Describing the governance of the 2020 COVID-19 outbreak in China from a sociopolitical perspective, we found that the approach of government had the following features. First, the Chinese government has developed a National Contingency Plan for Public Health Crises, in which, the public health crises are classified into especially significant (level I), significant (level II), major (level III), and general (level IV) crises according to their nature, hazard rating, and scope of influence (Jiang and Zeng 2021). Different decision-making bodies are designated for different levels of crisis: the decision-making body for level I crises is the State Council; that of level II crises is the relevant provincial government, which shall issue relevant emergency orders to subordinate units; that of level III crises is public health crisis response administration(s) above the prefecture level (counties directly under the provincial government); and that of level IV crises is the public health crisis response administration(s) at the county (city and district) level.

Upon the outbreak of COVID-19, the Hubei Provincial People's Government initiated A level II crisis response; after that, many local governments initiated level I crisis responses; on 25 January 2020, the Standing Committee of the Political Bureau of the Central Government, China's highest leadership body, set up the Central Leading Group for COVID-19 Prevention and Control (中央应对疫情工作领导小组), which, under the in-person guidance of General Secretary Xi Jinping, issued a series of important instructions, and put forward the general "United to Take Science-Based and Targeted Measures and Fight the Epidemic with Confidence" directive, with 
priority given to the safety of people's lives and physical health, sending a positive political signal (Xinhua News Agency 2020b). The establishment of a Central Leading Group by the central government means that the latter is taking over and coordinating the governance of the crisis. After the establishment of the Central Leading Group for COVID-19 Prevention and Control, Vice Premier Sun Chunlan was designated to head the group, coordinate and solve the difficulties of local governments in the allocation of materials and personnel, and play a key role in the construction of the two major infectious disease hospitals and the Fangcang hospitals (temporary treatment centers). She was also tasked with investigating officials for inaction, and responding to people's social concerns (People's Daily Online 2020).

Chinese governments at all levels have been reorganizing their departments horizontally to improve their crisis response capabilities. The State Council of China has twenty-six ministries and departments, each with independent administrative functions, which often results in information asymmetry and poor cooperation and coordination between departments. The Central Leading Group for COVID-19 Prevention and Control under the State Council and its subordinate departments closed up the original sector segmentation by setting up cross-department working groups, such as the Comprehensive Group, Medical Relief Group, Medical Material Support Group, Living Material Support Group, Scientific Research Group, and Expert Group, according to the needs of epidemic prevention and control. Each group is composed of members from multiple departments (as shown in Figure 4). Take the medical material support group as an example: it contains members from the Ministry of Transport, the National Health Commission, the Ministry of Civil Affairs, and the Ministry of Finance. The member units unify in times of crisis to set prevention and control objectives, dispatch and allocate resources, implement prevention and control measures, provide medical assistance, and release information (Gao and Zhang 2020). All levels of government also follow the central approach to establish an integrated command center to coordinate the allocation of resources across departments. Leading groups (领导小组机制) are a unique organizational form in China's political system: they can be characterized as task-oriented, ad hoc groups that handle special issues by special means; they are flexible, able to break down procedural frameworks and centralize the resources required for solving immediate problems. The leading group mechanism, to a large extent, breaks down the information silos of different departments, and thereby improves the efficiency of resource allocation, which is the most important issue during public health crises.

In addition, China has also strengthened its vertical accountability mechanism and thereby improved the efficiency of its epidemic prevention organizations. China has four levels of government: provincial, prefectural, county, and subdistrict/township. Coordinating officials at all levels of government to act in unison is critical to crisis governance. The Chinese government operates differently from the typical Western bureaucracy: intergovernmental responsibility contracts are signed between all levels of government and administrative matters are subcontracted to the grassroots orga- 


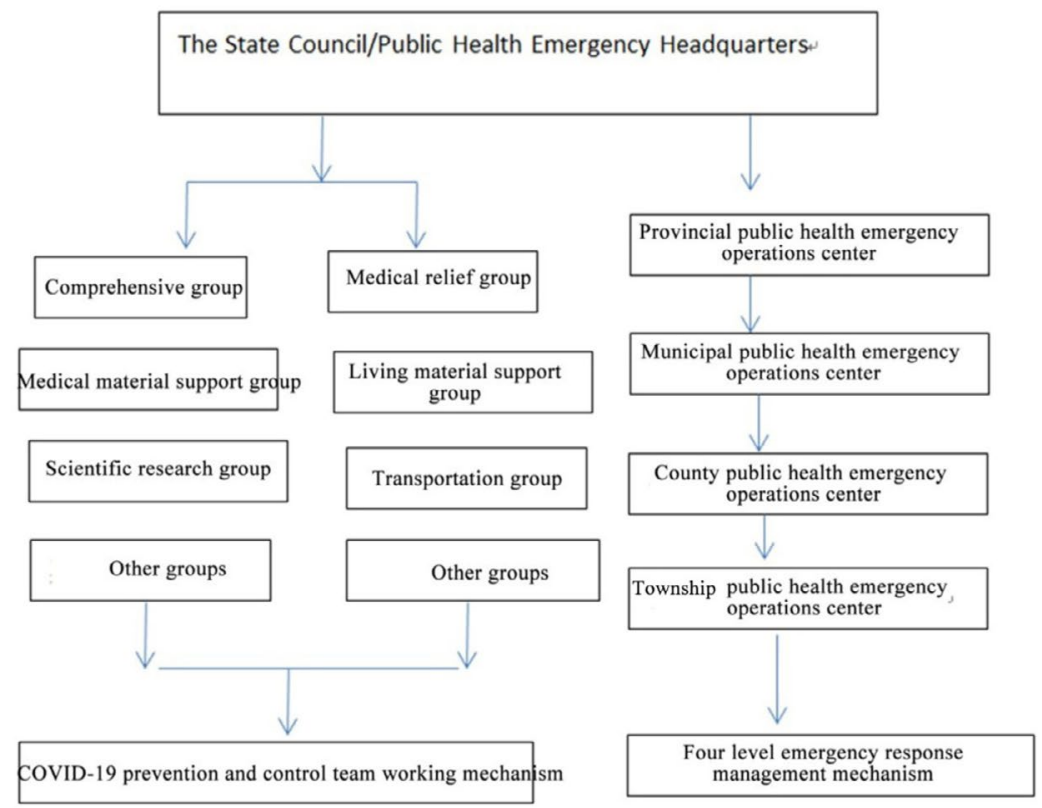

Figure 4. The Chinese Government's Response System to COVID-19 (Source: National Health Commission of the People's Republic of China)

nizations. Superior governments usually implement promotion incentives, especially in the case of a major public safety crisis: leaders and cadres who make outstanding contributions are more likely to be noticed and promoted and given important responsibilities in the administrative system more quickly. In contrast, public officials who fail to perform their duties will face various disciplinary actions such as warnings, demerits, demotions, dismissals, and expulsions. This accountability-based incentive was widely used in the fight against COVID-19, and according to the relevant statistics, the number of people held accountable during the fight against this epidemic was about three times as high as that during the SARS outbreak (Han 2020).

In the early stages of the anti-epidemic work, central government agencies effectively alerted and mobilized leading cadres via accountability mechanisms to ensure the execution of precise prevention and control measures-all while responding to public concerns and reassuring Chinese citizens in a timely manner. In addition to the implementation of these measures, the agencies' work revealed how well the various local governments responded to the crisis amid intense public pressure. The local cadres and grassroots cadres that performed responsibly and efficiently while working on the frontlines saw many of their members receive promotions.

The nationwide coordination of resources was carried out with priority given to key regions. At the peak of the epidemic, due to the limited resources under its direct control, the central government implemented a targeted assistance mechanism 
(对口支援机制), issuing administrative orders to twenty-nine provincial units to form medical teams to support Wuhan, which was followed by a request to nineteen provinces to provide targeted (human, material, and financial) assistance to sixteen other municipal administrative units in Hubei Province under the slogan "one province for one city." Support units divided up their responsibilities in the assistance mission under the principles of "one province supports one city" and "one city supports one county." Supporters and recipients communicated and consulted with each other with regard to the sending and receiving of medical supplies (including preventative equipment) and living materials.

Targeted assistance is an institutional arrangement in China with a long history. There was a long period of time when developed eastern provinces and cities in China supported less developed western regions. These provinces' support efforts were taken into account by the state in their internal performance assessments in order to ensure that such efforts were successful. The targeted assistance implemented during the fight against the COVID-19 pandemic is such an example of cross-regional resource reallocation and benefit redistribution among lower levels of government under the oversight of the central government and can be considered as a kind of horizontal transfer payment (Shi et al. 2020). In the context of traditional Chinese culture, the relation among provinces and cities is described as one between siblings and/or neighbors. This culture of mutual assistance makes supporters convert their politically mandated aid mission into a moral obligation to help their compatriots overcome difficulties. Due to media coverage and the public's strong approval of mutual support, various entities may sometimes even compete with one another to offer support in times of crisis. If they are successful in providing aid, they will receive positive coverage and public adulation, but if they fare poorly, they will receive scorn. Therefore, it comes as no surprise that the best medical resources from Peking Union Medical College Hospital, Xiangya Hospital of Central South University, Qilu Hospital of Shandong University, and West China Hospital Sichuan University all gathered in Wuhan during the pandemic.

\section{The Sociocultural and Welfare Dimension: The Dynamics of Community Involvement}

The rapid horizontal spread of the COVID-19 pandemic required early detection of the pandemic at the community level in order to break its transmission chain. Thus, the Chinese government organized extensive and sufficient societal grassroots mobilization, reflecting the advantages of the Chinese "whole nation system." The so-called "whole nation system" is designed to mobilize all possible forces under the leadership of the central government to form a nationwide actor network to deal with crises ( $\mathrm{He}$ 2020). The community actor network includes community workers, societal organizations, volunteers, party groups, community health service personnel, police officers, 
property management personnel, residents, and all other societal forces that can be organized and mobilized. The extensive and intensive societal grassroots mobilization, by making full use of the advantages of the strength of communities, was the basis for China's ability to curb the spread of COVID-19 pandemic rapidly and effectively. Surveys carried out in China demonstrate that the trust of people in the politics of the government is high. According to three rounds of data collection by the Social Mentality Research Team of Harbin Engineering University from mid-February to early March 2020, more than 80 percent of respondents felt that "government departments" played the most significant role in the fight against the epidemic, and more than 80 percent of respondents felt safer (Zhao et al. 2020). Various strategies were deployed to involve people and create the community participation that was so crucial to the COVID response.

\section{The Grid Clerks Management Approach}

During an epidemic, the community becomes the frontline of epidemic prevention and control, and community autonomy and the spirit of mutual support and solidarity play a major role during the outbreak. The community is divided into a number of grid squares according to certain criteria based on the original village community structure, and the grid clerks in each square are responsible for collecting and providing feedback on information gleaned from the area as well as for channeling and coordinating the information through the coordinating body.

Since the work of grid clerks consists of informing people about government policy and information gathering, they were put into the field to help carry out China's COVID-19 pandemic response as a means to help stop the spread of the disease. In the early stage, members of the public knew little about COVID-19. They did not pay enough attention to it and did not know how to protect themselves on the basis of the scientific information available to them. The grid clerks, however, went to the villagers' homes to spread the knowledge of pandemic prevention and explain to the villagers how to wash their hands properly, wear masks correctly, and keep indoor air circulating well. They also carried out online awareness campaigns on various social media platforms, including WeChat groups and Moments, and updated the public, in a timely manner, on the latest progress made by the state, making them propagandists for China's pandemic prevention efforts.

Another most important task that these clerks have is to carry out a thorough investigation of the settled and itinerant populations in each grid square. In this case, they had to screen people returning to rural areas from Hubei Province and people with red and yellow health codes, paying attention to the dynamics of key areas and key populations, and reporting the progress of their investigations every day. This greatly improved the quality and efficiency of the pandemic investigation work. In addition, the grid clerks needed to pay attention to societal conditions and public opinion in a timely manner, as they reflect the vital needs of the population at large, 
and they also participated in the mediation of conflicts and disputes caused by the pandemic in order to help assuage people's worries and concerns.

In addition, together with the grassroots workers, the grid clerks also shared the tasks of purchasing and delivering supplies, community access control, and temperature measurement and registration. During the critical period of epidemic prevention and control, community grid management contributed to the effective implementation of the home quarantine policy. Therefore, the grid management approach takes full advantage of community member autonomy. Organizing idle labor in the community (e.g., married women working locally) not only provides a direct way for citizens to participate in pandemic prevention and control, but also facilitates the smooth bottom-up communication of citizens' needs so as to better serve people (Tao et al. 2020). The information about family conflict, strangers coming to the village, and other new items that are easy to inquire about in village society will be quickly communicated to the grid clerks, and they need to rush to the scene for processing or verification as soon as possible. For the grid clerks, their work attributes require them to pay more attention to the living areas they are familiar with, make close connections with individuals and the community, and make themselves "busybodies." More profoundly, various part-time grid clerks and volunteers have established an interaction network among people within the community after acquiring the information on the basic societal conditions, basic interpersonal relationships, and people's lives in the community, thus helping communities improve their own governance (Tao et al. 2020). All of these tasks are conducive to the improvement of social cohesion and the construction of collective identities. And this is one reason why China's efforts to combat COVID-10 were successful.

\section{Societal Organizations and Volunteer Groups}

Since the "first large-scale collective appearance" of Chinese nongovernmental organizations (NGOs) in 2008 (Zhu et al. 2009), societal-oriented organizations have repeatedly played an important role in daily life and in public crises because of their professional attitude toward work and their espousal of such values as respect and equality. These organizations actively and successfully seek possible action spaces outside the official functions of the government (He and Sun 2020). In recent years, both local governments and grassroots community representatives have shown a great deal of appreciation for the important role that these organizations play in new forms of local governance. Studies demonstrate that the participation of these organizations can greatly enhance the professionalism of grassroots groups when it comes to local governance processes and can give community governance a strong social component (Tian 2020).

Societal organizations and volunteer forces are also important participants in pandemic prevention. Because these organizations are closer to people's needs, their response speed and flexibility are better than those of the bureaucratic system. Many 
organizations (e.g., various foundations and volunteer groups) raised money globally to help medical facilities that lacked protective supplies (Tang and Wu 2020). Compared with others, volunteers are more attentive to the urgent needs of disadvantaged groups such as elderly persons with no family, children in quarantine, and people with disabilities, and actively provide them with psychological support and assistance. According to one source, there are more than 600,000 volunteers in Hubei Province working on the frontline of epidemic prevention and control (Yao 2020).

Due to the requirements for COVID-19 prevention and control, the participation scale of societal organizations is not as large as it was during the Wenchuan Earthquake in 2008, but the joint action tradition of these organizations continues. Outcomes of studies demonstrate that large-scale "online and offline" joint actions of social organizations have improved information sharing, resource links, community pandemic prevention, and services for special populations (Yang et al. 2020). On 2 February 2020, the Amity Foundation and the Narada Foundation, together with other foundations, jointly established the Social Organization Cooperation Network Fighting against the COVID-19 Pandemic, which became the most influential cooperation network in the early stage of the pandemic. Its main objectives were to share action information, evaluate the requirements for pandemic prevention, disseminate public health knowledge and support relevant capacity-building, establish an international information coordination mechanism, share international expertise, and even promote international joint action. In addition, the China Social Entrepreneur Foundation (CSEF), together with the Zero Zero One Rescue Team and other societal organizations, launched the Joint Action fighting against the COVID-19 Pandemic, whose methodology is based on the disaster relief approach used during the Wenchuan Earthquake (Yang et al. 2020). All members, by making full use of their core advantages in resources, technology, and procurement in their respective fields, raised and donated urgent strategic materials and provided professional volunteer services for areas affected by the COVID-10 epidemic.

\section{The Socioeconomic and Financial Dimension: Mobilizing Economic Resources}

\section{Industrial-Economic Foundations for Prevention, Control, and Support}

Just like other countries in the world that lacked epidemic prevention materials during the high prevalence period of COVID-19, China also faced shortages of medical supplies during the early stage of COVID-19 outbreak. To cope with this deficit, the Chinese government mobilized medical supply producers to resume work and production and participate in the production and supply of epidemic materials; from hospital reconstruction and production line preparation to the production and development of melt blown cloth, masks, protective clothing, and testing reagents, enterprises in all 
sectors provided a lot of support for the production of epidemic prevention materials during the early stage of the epidemic, regardless of the cost. By April 2020, China's domestic medical supply production basically met the domestic demand for epidemic prevention materials, of which, the daily production of medical protective clothing and medical N95 masks reached 800,000 units and 5 million units, respectively; the daily output of instant hand sanitizer reached 308 metric tons; and the daily output of automatic infrared thermometers reached 3,400 units (CBIRI 2020). The capacity expansion of epidemic prevention materials has laid a solid material foundation for epidemic prevention and medical equipment manufacture.

Chinese producers of epidemic prevention materials have resumed work and production relatively quickly, thanks in large part to the industrial support policies from local governments. These policies mainly include tax and fee reductions, policy-based finance, special funds, job stabilization measures, and public service support (Gao and Shen 2021). We will not look at each of these items in turn.

\section{Tax and Fee Reduction}

When enterprises have tight capital flows, making it harder for them to do business, the state can help them out by reducing various taxes and fees: this helps reduce costs and expenditures, and frees up funds that can be invested back into the business. During the pandemic, local governments active tried to exempt enterprises from having to pay the Value-Added Tax (VAT) on income generated from transporting key security materials for epidemic prevention and control and from providing public transportation services, living services, and postal express services. Measures to reduce, return, and defer insurance premiums were also enacted: from February to December 2020, the fees for basic pension insurance, unemployment insurance, and work injury insurance for small and medium-sized enterprises were waived, while they were halved for large enterprises. In addition, 50 percent of the actual unemployment insurance premiums paid in the previous year were returned to participating enterprises that had no layoffs and relatively few layoffs.

\section{Policy-Based Finance}

Policy-based finance measures mainly include subsidized credit and credit guarantees. Many provinces in China, such as Guangdong, Hunan, and Anhui, have implemented a subsidized credit policy, and the key enterprises tasked by the state and provinces to participate in epidemic prevention enjoyed preferential interest rate credit support: this means that the interest rate ceiling did not exceed the one-year LYR minus 100 basis points. Based on supporting financial subsidies, the actual financing cost of relevant enterprises was reduced to 1.6 percent or lower. With the special refinancing policy of the People's Bank, Zhejiang Province, for example, gave out financial subsidies on loans to key enterprises participating in epidemic prevention and control; for eligible enterprises, the government subsidizes 50 percent of the actual interest rate of the loan for a period of not more than one year. The subsidized credit policy, 
then, eased the financial burden on various enterprises during the first stage of the pandemic.

\section{Special Funds}

The local government supports key enterprises in the production of epidemic prevention and control materials, encouraging them to expand and increase their production of important medical prevention and control materials in short supply. All the excess medical prevention and control materials produced by these enterprises are purchased and stored by the government. Provincial key manufacturers of emergency supplies that rapidly expand production and capacity and produce urgently needed epidemic prevention and control materials through "quick" technological transformation and equipment replenishment are eligible to receive financial support when they suffer losses or invest in equipment during the epidemic prevention and control period. Enterprises that contribute to the supply and security of supplies for epidemic prevention and control can receive certain incentives as well. The government has set aside special funds for the informatization and digitalization of industries, uses incentive funds for the creation and development of high-quality manufacturing technologies, and uses industrial funds to support for companies in such leading-edge areas as robotics, big data, and artificial intelligence. The government also invests funds into medical science research so as to increases the research and development of original new drugs, medical supplies, medical devices, and precision diagnosis and rapid testing equipment. In order for enterprises to undertake the emergency research and develop projects for epidemic prevention purposes (projects initially designed by the province), the government will fund the projects' startup costs and provide an initial round of funding according to the "special case" principle; it will then give these companies subsidies based on their performance (Gao and Shen 2020).

\section{Job Stabilization}

Maintaining job stability and ensuring balanced supply and demand in the labor market are effective measures that were taken by the government to ease the pressure on enterprises during the COVID-19 epidemic. Local governments helped enterprises to quickly resume recruitment, work, and production through measures such as transportation subsidies and job subsidies. The Anhui provincial government proposed that all cities and counties use employment subsidy funds to support key enterprises for prevention and control material production by giving one-time employment subsidies at the rate of RMB200 per person per day. In Zhejiang Province, the Huzhou City People's Government went a step further. It issued the Opinions on Subsidy and Reward for Enterprise's Work and Production Resumption, a decree clearly stipulating that a subsidy and reward fund of no less than RMB100 million would be set up for the resumption of work and production at local enterprises (be they industrial, service, or construction enterprises). Newly recruited employees would each be given a one-off living subsidy of RMB1,000, and anyone who was to bring twenty people or more to 
Huzhou City for employment would be given a one-off reward of RMB200. Furthermore, if an enterprise were to organize vehicle transport for employees living outside of the city, the related fees would be fully paid by the city. The city government's job support measures gave companies an incentive to quickly organize their activities and get their employees back to work (Gao and Shen 2021).

\section{Public Service Support}

During the epidemic, people in China, like elsewhere, were very worried about the disease and about their health, and it was not enough for enterprises on their own to promise workers that they would be safe. Local governments needed to accurately assess the development of the epidemic, take the initiative to provide enterprises with information on the epidemic, and help the latter with public health campaigns. In this regard, Zhejiang Province pioneered the Five-Color Map of the Epidemic (i.e., the Epidemic Risk Map of Zhejiang Province). Based on four assessment indicators, including total confirmed COVID-19 cases in each county (cities and districts), proportion of local cases, aggregated outbreaks, and absence of new confirmed cases for three consecutive days, Zhejiang Province rated the risk level of the epidemic in 90 counties. There were a total of five levels — high, higher, medium, lower, and low-that were correspondingly red, orange, yellow, blue, and green on the map. Zhejiang took the Five-Color Map of the Epidemic as an effective way to implement safety protocols: the counties labeled "high" and "higher" should take epidemic prevention and control as their top priority and strictly implement the so-called "eight control mechanisms"; the counties labeled as "medium" should adhere to the priority of epidemic prevention and control and promote work resumption safely and in an orderly fashion; and the counties labeled as "lower" and "low" should try their best to promote work resumption in the context of epidemic prevention. In addition, the governments of Zhejiang Province put forward the resumption rate targets for the province's counties until 23 February: the work resumption rate for green areas (low risk) should reach 70 percent and above, and the rate for blue areas (lower risk) should reach 60 percent and above.

From the perspective of experience and lessons learned in the fight against the epidemic, China's independent and complete national economic system and its strong industrial emergency response capability provided important support for the fight against the epidemic, while the complete industrial chain played a leading role in the fight against the epidemic in terms of material support, industrial emergency response, crisis response, and economic stability.

\section{Technological Development (IT) for Prevention and Control}

In the course of China's rapid economic development, Chinese internet technology companies have taken off rapidly: for example, Alibaba and Tencent have become the global leaders in technology development, logistics, and distribution. A distinctive feature of internet businesses is the platform economy, which gathers large numbers 
of users on a platform so as to form economies of scale. The Alipay platform developed by Alibaba has over 900 million users worldwide; Tencent's platform has over 1 billion WeChat users. During the epidemic, the private sector took advantage of the platform and scale model to build the Health Code Check APP for the public sector. In February 2020, Hangzhou City, Zhejiang Province, took the lead in exploring the dynamic management approach using the "red, yellow, and green" health code model. First, the individual authenticates their real name and fills in their health information on the smartphone applet, and the platform verifies the information filled in by the individual by comparing it with relevant data such as cell phone roaming data and close contacts, and then generates a QR (Quick Response) code (Shi and Ma 2020) in one of three colors. A green code enables its holder to move about unrestricted within the city. Someone with a yellow code may be asked to quarantine themselves for seven days. And red means that the individual must enter quarantine for fourteen days. After the quarantine period is over, the code will turn green (as shown in Figure 5). The health code has now been rolled out nationwide and has an established mutual recognition mechanism and a "one health code only" system. Compared with the traditional manual filling in of information and repeated information review, the health code improves efficiency and simplifies the management process, and, more importantly, it turns static data into dynamic data: the color of the code can be changed at any time according to the real-time dynamics of the individual's travel record and place of residence. After a sporadic outbreak of COVID-19, the health code allows the government to precisely locate the streets and communities where cases have been confirmed. The code turns yellow or red for those who have been to or have been living in such places, thus implementing precise epidemic prevention and control measures with minimum impact to people's everyday lives and to companies' everyday operations.

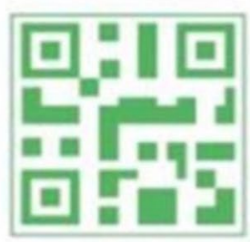

[Green code]

Pass with code

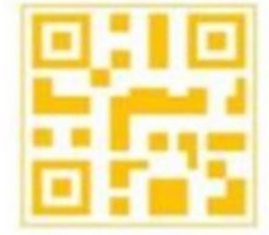

[Yellow code]

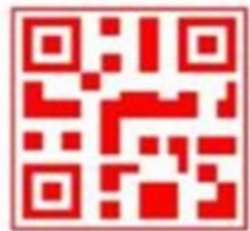

[Red code]

Red code means a 14-day quarantine, after that, the code will turn green if the holder's health status is normal during such 14 days

\section{Fighting COVID-19 We Are Together}

Figure 5. Hangzhou Health Code Rules (Source: https://baike.baidu.com) 
The application of the health code in China was successful owing to the interaction between the government and the country's enterprises. The health code was first applied in Hangzhou. Before the outbreak, Hangzhou had already invested a lot in the construction of its so-called "City Brain." "City Brain" is the core of smart city construction, integrating "small data" from the government, enterprises, and society into a "big data" system. The Hangzhou "City Brain" project was built by the Municipal Data Resources Management Bureau, which was newly established in 2019, with the cooperation of the Hangzhou Municipal Health Commission, Hangzhou Traffic Bureau, Hangzhou Public Security Bureau, Hangzhou Civil Affairs Bureau, and several technology companies. After the COVID-19 outbreak at the end of 2019, the "City Brain" changed the status of daily city operations into "combat readiness," and the City Brain COVID-19 Prevention and Control System was launched immediately. The Hangzhou Municipal People's Government asked all departments to use the City Brain Digital Cockpit to ensure the timely detection and effective handling of cases. The data from the above-mentioned health code app was interconnected with the data collected by the various city departments, and a unified digital declaration platform was established in the city to realize timely information-sharing and real-time case-tracking, forming an exhaustive and full-coverage prevention and control system. The efficient use of the health code in Zhejiang and even the country as a whole has effectively relieved the two major pressures on the state and society, that is, epidemic prevention and work resumption (Guo and Gu 2020).

\section{Economic Development as Reinforcement of Healthcare Capacities}

Over the past twenty years, China's rapid economic development has been accompanied by increasing government investment in the health sector, with total national health expenditures reaching RMB6,519.6 billion in 2019, accounting for 6.6 percent of GDP. This includes government health expenditures of RMB1,742.85 billion, accounting for 26.7 percent; social health expenditures of RMB2,927.8 billion, accounting for 44.9 percent; and personal health expenditures of RMB1,848.95 billion, accounting for 28.4 percent (NHCPRC 2020). In 2019, the total number of healthcare facilities reached $1,007,545$, an increase of 7 percent in ten years. The number of beds in medical facilities nationwide was $8,807,000$, of which 72.5 percent were in public hospitals. The number of beds per 1,000 persons grew from 3.42 in 2010 to 6.29 in 2019. From the composition of medical and nursing personnel, according to the available statistics, the number of licensed physicians per 1,000 persons increased from 1.79 to 2.77; the number of registered nurses per 1,000 persons went from 1.52 to 3.18 ; and the number of professional public health institutions per 10,000 persons increased from 4.76 to 6.41 .

These increases in infrastructure and human resources effectively guaranteed the efficient allocation and supply of medical personnel during an outbreak. At the early stage of the COVID-19 outbreak, Hubei Province, which was at the center of the 


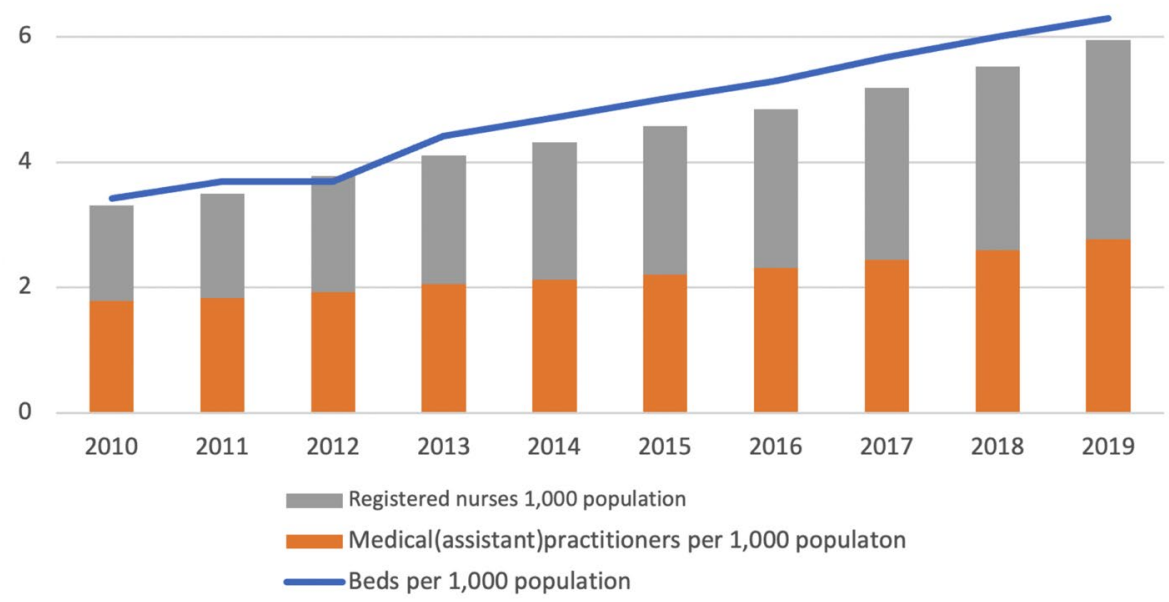

Figure 6. Changes in the Number of Chinese Health Technicians and Beds of Health Institutions during the Last Decade (Source: National Health Commission of the People's Republic of China [http://www.nhc.gov.cn/])

epidemic, faced the problem of an insufficient supply of medical personnel. The central government dispatched medical resources from all over the country to meet the needs of the center of the epidemic, and a total of 42,600 medical personnel from 346 medical teams were mobilized from all over the country for anti-epidemic assistance. Such a large medical workforce needs to rely on an established institutional system and human resources to support it. The strong supply of medical resources ensured that localities could respond quickly to the needs of sick populations and flatten the curve of outbreaks quickly, and this helped mitigate the epidemic nationwide.

The development of the healthcare sector has also provided fundamental support for regular outbreak prevention and control, and the rapid nucleic acid testing of large populations. China has adopted a strategy of mass nucleic acid testing in the routine governance of outbreaks, usually organizing mass nucleic acid testing in areas where confirmed cases have been located, or where confirmed cases have traveled. In the early days of the Wuhan outbreak, the city first used what is referred to as "batch testing" or "pool testing" on 11 million people in the city over a period of more than ten days, testing 1.47 million people each single day. In June 2020, cold-chain-imported food infections were detected in an agricultural products market in Beijing, and the Beijing Center for Disease Prevention and Control tested half of the city's population based on epidemiological survey data, completing a total of over 11.88 million nucleic acid tests. In October 2020, Qingdao City, Shandong Province, experienced in-hospital cross-infection and implemented city-wide nucleic acid testing for 10.82 million people to avoid the spreading of the virus throughout the city. As of 31 May 2020, a total of RMB162.4 billion had been arranged at all levels of finance for epidemic prevention 
and control, and 58,000 patients (confirmed cases) had been discharged from the hospital. The medical expenses totaled RMB1.35 billion, and the amount of per capita medical expenses was about RMB23,000. Over the past year, despite multiple sporadic outbreaks and local cluster cases, none of them has developed into large-scale epidemics, and the number of confirmed cases and rebounds has been controlled to a limited scale, which is impossible without mass nucleic acid testing and enhanced epidemiological surveys carried out efficiently by local governments (Bradsher and Buckley 2020).

The steady development of the health sector has improved China's resilience in handling public health crises. First, it helps governments at all levels to transfer health resources horizontally, alleviating the crisis in disaster centers and mitigating risk quickly. And second, during the epidemic control normalization period, high-quality public health facilities and health-related human resources were indispensable for local health departments to be able to carry out large-scale detection and identification to effectively prevent outbreaks.

\section{Conclusion}

This article describes the governance characteristics of China in response to the COVID-19 outbreak from a sociopolitical, socioeconomic, and sociocultural perspective. From a sociopolitical perspective, the government adopted a whole of government approach. Basically, it is a top-down, centralized governance approach led by the central government. A series of unique institutional arrangements were implemented in a short period of time. A central governmental Public Health Emergency Group was established to take the lead. Segmentation between policy departments was broken down and rapid responses were organized to meet local emergency needs. Vertical accountability mechanisms and measures punishing and promoting certain behaviors have largely motivated local officials to mobilize resources and deliver results. Societal agencies and other tools were realized to involve the public at the grassroots level. Counterpart support mechanisms were launched. The extensive and active centralized interventions facilitated the extraordinarily efficient containment of the epidemic.

From a sociocultural perspective, the government was fully committed to mobilizing and motivating forces at the grassroots level. Local governments, community grids, volunteer agencies, and other societal organizations were deployed to involve communities at the grassroots level. As a result, numerous ordinary people actively participated in the frontline volunteer service, which promoted active citizen participation. In surveys, people showed high levels of appreciation of the government's measures. Full use was made of the strengths of community organizations. Through this powerful approach, the transmission paths of COVID-19 were effectively cut off and societal stability was achieved.

From a socioeconomic perspective, the independent and complete national economic system was mobilized to provide support for combatting the pandemic. The supply 
of epidemic prevention materials and the chain of medical materials were rapidly guaranteed. The ongoing economic development has provided the resources for the improvement in the quality of healthcare services (e.g., the effective allocation of medical personnel during the outbreak). Thus, mass nucleic acid testing and universal vaccination were guaranteed. Economic development also guaranteed that the state's finances could bear the burden of the related costs. Medical insurance and critical illness insurance ensured that COVID-19 patients got treatment. Through universally accessible information technology provisions, advanced epidemic prevention tools were introduced. QR codes were used as a tool to quickly identify and control individuals with health risks. The data, assembled by this tool, facilitated epidemiological investigations and the further development of risk prevention and control.

In our study, it has become clear that the sociopolitical, sociocultural, and socioeconomic dimensions strongly do interact with one another. Though mainly described from the sociopolitical perspective, the reciprocal processes and events that occurred during the pandemic do influence and reinforce each other. The comprehensive intersectoral understanding of what was going on was clearly demonstrated.

During the COVID-19 pandemic crisis, characteristic features of the "societal" dynamics of countries are uncovered. The governance approach of each country stems from, among other things, their particular societal (historical, political, economic, and cultural) background. It may, for instance, be strongly centralized, and it may be strongly decentralized, and it may even be both at once. Besides numerous other factors (e.g., socioenvironmental, demographic, sociogeographic, sociocultural, and socioeconomic), a country's practiced governance approaches contribute considerably to the results of epidemic control efforts. It therefore would be prudent to conduct comparative studies to trace and explain significant differences, though in these hectic epidemic times such in-depth studies can be complex.

From our description of the governance approaches in China, and the involvement and responses of the public, societal agencies, and companies, such questions deserving further investigation do arise. As an example, an important issue that came forward concerns the precise nature of the processes related to community participation. The participation of people and communities can be interpreted in each of the three social quality dimensions. From a sociocultural and welfare perspective, it means that people show the willingness and are prepared to unite, cooperate, and respond generously to initiatives from outside (e.g., government, organizations) to meet their welfare and cultural needs. From a socioeconomic perspective, people do unite, act, or respond because of their own or others' economic interests. From a sociopolitical perspective, people purposely are "socially empowered' to participate and express themselves in processes of political decision-making. Shedding light on the right interpretation of what "community participation" means requires more in-depth (and comparative) analytical studies.

In our study, we have limited ourselves to describing the structure, processes, and immediate effects of a multitude of governance approaches initiated and controlled by 
the central government. We have not attempted to get a better and deeper understanding of the precise intentions and "social" relationships of the processes of governance that we have depicted. We have not analyzed the quality of the "conditional factors," and we did not include the personal perceptions of people about these conditions as "constitutional factors." After all, the dialectical processes between these two sets of factors precisely (re)produce a particular degree of "social quality." Also, we did not attempt to include elaborations of the "normative factors" of social quality, which would enable us to ethically evaluate what has been going on. However, our descriptive study did provide the opportunity to identify some interesting questions related to the social quality of Chinese societal dynamics.

SQT and the SQA—from their conceptualizations of "the social," "social quality" to their "comprehensive analytical architecture" — are seen by us as the framework of choice to identify and elaborate the questions that we want to raise. These questions could be taken up with regard to the COVID-19 pandemic, to other critical situations, and to post-pandemic issues. We would like to conclude our study by listing some of these questions.

An insufficiently covered area in research is how (objectively assessed) conditional factors relate to (subjectively assessed) constitutional factors. Referring to the pandemic, the question would be: how do the "objective" nature, structure, workings and quality of the societal conditions (created by the government and other societal institutions) relate to "subjective" perceptions of these conditions by the people concerned.

Regarding this question, interesting studies, based on the 2017 National Chinese Social Survey, were published in this journal. Li Wei and Cui Yan, applying the conditional factors analyzed the quality of people's daily circumstances. Notwithstanding the impressive improvement of socioeconomic security in China, the level of social security appeared far behind the actual needs of the population:

Many respondents believe the current level of social security is too weak to serve as a guarantee, and they value the guarantee low. At the same time, although the disparity between the urban and rural areas in terms of socioeconomic serious entitlements continues to narrow, the level of social security for rural residents is still far lower than that of urban residents ... When the "relationship" and "background" become decisive factors affecting individuals' development in society, social equality and fairness would be greatly undermined . . . the data also show that respondents' sense of inequality is most pronounced in the areas of income distribution, as well as rights and entitlements between urban and rural areas. Whether we could further build a society with equal opportunities and homogeneous treatment is really relevant to our improvement of the quality of Chinese society in the future ... many respondents also show a great indifferent attitude toward political engagement. This situation indicates that the current level of social empowerment in China is relatively low because of [a] lack of political efficacy. (Wei and Yan 2018: 99)

Cui Yan and Huang Yongliang in another study argue that the Chinese society has entered a new stage of development. The main needs and demands of the population 
have gradually changed. They recognized new aspects of different layers or "social classes' in China and the change of the four conditional factors of "social quality" between these classes. An interesting conclusion is that the societal construction lags behind the economic construction:

In the current society, people face a decline in awareness of being governed by law, and lack values and moral restraints. Because the upper-middle group has failed to largely expand its channels of sociopolitical participation, this has produced a negative effect on the evaluation of the upper-middle group in society, to some extent, has shown a sense of "insufficiency of powers and functions and "political indifference" . . the existing income distribution system requires further improvement and adjustment, so people of the upper-middle and low income groups may develop a sense of deprivation in the income distributions, which would lead to the low evaluation of fairness and equality. (Yan and Yongliang 2018: 65)

In judging the outcome of the specific approaches of governance during the pandemic, the "social" structure and dynamics of Chinese society needs to be taken in account. It is argued, that, among other things, the government and societal organizations, by involving communities, can greatly enhance "professionalism" at the grassroots level. It is assumed that this leads to processes in which communities are given stronger motivations to participate in local governance (Tian 2020). Such an argument interprets "community participation" as a set of processes in the sociopolitical sense. These processes could, however, also be interpreted from the sociocultural or socioeconomic perspectives. Such interpretations would then of course reveal other meanings.

Intriguing questions, it seems to us, do arise from these considerations. They are related to the balances in the interrelationships and influences of the sociocultural, sociopolitical, and socioeconomic processes. Forces from these spheres to a large extent do determine the way "the self-realization of people and the formation of collective identities" are achieved. They equally do concern the balance between the primacy of "the individual" and "the collective." The latter, (individualism versus collectivism) of course, is an immanent subject of sociopolitical debate (Ang 2020; Dodds et al. 2020; He et al. 2020).

This subject also concerns our heuristic example of community participation. The question is: should "participation" be interpreted as individuals and their communities being subjects-being part of the whole of government approach - who primarily serve as contributors to collectivities, or should these processes be interpreted as actions of the collective (the government) to empower residents as "political actors" prepared to participate. This question also relates to the nature of the forces in operation to determine the behaviors and self-realization of individuals. Are these mainly sociocultural, socioeconomic, and sociopolitical in nature? The question, in other words, becomes: by qualifying the "participation of communities and residents" from the above perspectives, how should the shortfalls and/or successes of different governance approaches to control the pandemic be interpreted? 
In this respect, China indeed may be an interesting case. It is a common assumption that collectivism in China's culture is highly valued. Dedication and self-sacrifice are common goods. The famous historian Sima Qian wrote in A Letter of Reply to $\operatorname{Ren} A n$ : "Though death befalls all men alike. It may be weightier than Mount Tai or lighter than a feather." It expresses a core value of Chinese culture: if the individual is dedicated to the collectivity or the country, the value of the individual's life will be maximized; otherwise, it is insignificant. The spirit of collectivism is one among others expressed in the idea that families and country have the same structure. The country is a big version and extension of the family. It is the muscle on which the family tissue survives. Families do flourish only when the country is prosperous; they will break down in a ruined country. It is a common (and justifiable) assumption that it is because of these common values that Chinese residents trust the government and follow the national prevention and control policies. The benign interaction between government, societal organizations, and citizens, stemming from this cultural tradition of collectivism, has created favorable conditions for China's fight against the epidemic.

By posing this assumption, the area for further research and debate is given. It relates to the balances and qualities of the dialectic processes between the realization of the self of individuals and the (re)production of collective identities leading to a certain degree of social quality. According to our assumption, in Chinese society the primacy lies with the formation of collective identities. In the cited literature, it is suggested that in societies based on neoliberal ideologies the primacy lies with the self-realization of the individual. The question becomes normatively loaded by including the balance of the interrelationships and influences of the sociopolitical, sociocultural, and socioeconomic forces that determine self-realization and people's behaviors. In other words, is the self-realization of individuals determined primarily by sociopolitical forces, or socioeconomic forces, or sociocultural forces? And finally, using the social quality normative criteria (social justice, solidarity, equal value, and dignity), how should the outcomes be valued?

We have not been seeking the answers to these questions in our study. However, to us it seems that these questions may hold interesting points of departure for comparative research between societies holding different sociopolitical, socioeconomic, and sociocultural traditions.

Wang Jing is the Deputy Director of and an Associate Researcher at Department of Social Work and Sociology of Welfare, Institute of Sociology, at the Chinese Academy of Social Sciences. In 2019, she received a Fulbright Program grant to visit and conduct research at Harvard University. In 2014, she received a grant from Germany's Bausparkasse to visit and work at the University of Mannheim. Her main research interests include social policy theory, services for and policies related to the elderly, and housing policy. Email: wangjingcass@126.com 
Wang Xue is a Master's Degree Candidate at School of Sociology, University of Chinese Academy of Social Sciences. Her research interests include social policy and social security, long-term elderly care, and the economics of healthcare.

Email:wangxue2@ucass.edu.cn

\section{References}

Abdou, A. M. 2021. "Good Governance and COVID-19: The Digital Bureaucracy Response to the Pandemic (Singapore as a Model)." Journal of Public Affairs e2656. doi:10.1002/pa.2656.

Ang, Y. Y. 2020. "When COVID-19 Meets Centralized, Personalized Power." Nature Human Behavior 4: 445-447. doi:10.1038/s41562-020-0872-3.

Beck, W., L. J. G. van der Maesen, and A. Walker, eds. 1998. The Social Quality of Europe. Bristol: Policy Press.

Beck, W. A., L. J. G. van der Maesen, and A. C. Walker. 2012. In Social Quality: From Theory to Indicators. London: Palgrave Macmillan.

Bouey, J. 2020. China's Health System Reform and Global Health Strategy in the Context of COVID-19. RAND Corporation CT-A321-1, 20 May. doi:10.7249/CTA321-1.

Bradsher, K. and C. Buckley. 2020. "In This Coronavirus Wave, China Tries Something New: Restraint.” New York Times, 19 June. https://www.nytimes.com/2020/06/19/world/asia/ coronavirus-china-beijing.html.

CBIRI (China Business Industry Research Institute). 2020. "Analysis on the Current Situation of China's Epidemic Prevention Supplies Market in 2020: The Production of Medical Protective Clothing Increased by 90.6 Times in 3 Months." [In Chinese.] Askci.com. 8 June. https://www.askci.com/ news/chanye/20200608/1144251161579.shtml.

Cui Yan and Huang Yongliang. 2018. "Social Quality of China”, International Journal of Social Quality, 8 (1), pp. 49-68, DOI: 10.3167/ijsq.2018.080104

Dodds, K., V. Castan Broto, K. Detterbeck, M. Jones, V. Mamadouh, M. Ramutsindela ..., and C. Y. Woon. 2020. "The COVID-19 Pandemic: Territorial, Political and Governance Dimensions of the Crisis.” Territory, Politics, Governance 8 (3): 289-298. doi:10.1080/21622671.20 20.1771022.

Gao, C., and M. Shen. 2020. "Training Materials for Cadres in Zhejiang Province." In 100 Best Cases to Promote Resumption of Work and Production (Volume I), 88-101. Hangzhou: Zhejiang People's Publishing House.

Gao, Q., and H. Zhang. 2020. "Organization and Operation of Emergency Command of Public Health Emergencies from the Perspective of Customary Law: Take the Sample of COVID-19 Prevention and Control Work Leading Group and Headquarters." [In Chinese.] Academic Exchange 5: 47-60.

Guo, F., and X. Gu. 2020. "The Construction and Improvement of State Surveillance Capacity: Reflection in the Context of Public Health Crisis." [In Chinese.] Journal of Public Administration 3: 6-26.

Han, Z. 2020. "The Operation Logic of 'Accountability' and Its Effects: A Case Study of the Fight against COVID-19.” [In Chinese.] Urban Governance Research 5 (1): 60-75.

He, X. 2020. "Community Governance in the Face of Viruses: Networks of Grassroots Actors in the Juguo System.” [In Chinese.] Social Development Studies 7 (2): 2-10. 
He, X., and X. Sun. 2020. "International Community Action Network to Prevent Imported COVID-19: Social Work Intervention of Social Organizations.” [In Chinese.] Hebei Academic Journal 40 (6): 164-169.

He, A. J., Y. Shi, and H. Liu. 2020. "Crisis Governance, Chinese Style: Distinctive Features of China's Response to the Covid-19 Pandemic." Policy Design and Practice 3 (3): 1-17. doi:10.1080/ 25741292.2020.1799911.

IASQ (International Association on Social Quality). 2019. Ideas and Suppositions about the Application and Elaboration of the "Social Quality Approach" (SQA) in Eastern Europe: The Case of Ukraine. IASQ Working Paper No. 17. https://socialquality.org/wp-content/uploads/ IASQ-Working-Paper-17-4.pdf.

Jiang, C., and M. Zeng. 2021. "Analysis of the Relationship between Emergency Classification and Emergency Response Classification." [In Chinese.] Chinese Public Administration 2: 131-136.

Kettl, D. F. 2020. "States Divided: The Implications of American Federalism for COVID-19.” Public Administration Review 80: 595-602. doi:10.1111/puar.13243.

Li Wei and Cui Yan. 2018. "Social Quality of China:Indicators, Reality, and Problems", International Journal of Social Quality, 8 (2), pp. 78-102, DOI: https://doi.org/10.3167/IJSQ.2018.080205

NHCPRC (National Health Commission of the People's Republic of China). 2020. China's Statistical Communique on Health Development 2019. http://www.nhc.gov.cn/guihuaxxs/s10748/202006/ ebfe31f24cc145b198dd730603ec4442.shtml (accessed 19 September 2021).

Novakova, Z. 2017. "Four Dimensions of Societal Transformation: An Introduction to the Problematique of Ukraine.” International Journal of Social Quality 7 (2): 1-29. doi:10.3167/ IJSQ.2017.070202.

People's Daily Online. 2020. "Log of the Leading Group for COVID-19 Response of the CPC Central Committee.” [In Chinese.] People's Daily Online, 4 February. http://www.qstheory.cn/ 2020-02/04/c_1125529482.htm.

Qian, Y., and B. R. Weingast. 1997. "Federalism as a Commitment to Preserving Market Incentives." Journal of Economic Perspectives 11 (4): 83-92. http://www.jstor.org/stable/2138464.

Ricceri, M. 2019. "The Contribution of BRICS to the Quality of Global Development." International Journal of Social Quality 9 (1): 1-31. doi:10.3167/IJSQ.2019.090102.

Shi, C., and L. Ma. 2020. "Collaborative Governance, Technological Innovation and Smart Epidemic Prevention: A Case Study based on "Health Codes'." [In Chinese.] Studies on Party and Government 4: 107-116.

Shi, X., L. Fan, and S. Shi. 2020. "Targeted Assistance in China’s Fight against COVID-19 from the Perspective of Public Economics." [In Chinese.]. Public Finance Research 8: 12-22.

Soon, S., C. C. Chou, and S-J. Shi. 2021. "Withstanding the Plague: Institutional Resilience of the East Asian Welfare State.” Social Policy Administration 55 (2): 374-387. doi:10.1111/ spol.12713.

Tang, H., and R. Wu. 2020. "Analysis on the Holistic Governance Approach to the COVID-19 Epidemic: A Practical Challenge and Optimal Choice." [In Chinese.] Journal of Hubei University (Philosophy and Social Science) 47 (3): 1-13.

Tao, J., W. Li, and H. Cao. 2020. "Two-Way Interaction: Logical Turn and Reflection of Grid Management at the Grassroots Level: A Case Study of Grid Management in Luqiao District, Taizhou City." [In Chinese.] Journal of Party School of Hangzhou Municipal Party Committee 1: 67-72. 
Tian, Y. 2020. "Construction of Urban Community Anti-Epidemic System from the Perspective of Governance.” [In Chinese.] Journal of Social Sciences 1: 19-27.

Van der Maesen, L. J. G. (2013). "Analysing Societal Circumstances, Sustainability and Sustainable Urban Development: New Theoretical and Methodological Challenges for Social Quality Indicators'. International Journal of Social Quality, 3 (1), pp. 57-82, https://doi.org/10.3167/ IJSQ.2013.030106

Van der Maesen, L. J. G., and A. Walker, eds. 2012. Social Quality: From Theory to Indicators. London: Palgrave Macmillan.

Westbroek, J., H. Nijhuis, and L. van der Maesen. 2020. "Evolutionary Thermodynamics and Theory of Social Quality as Links between Physics, Biology, and the Human Sciences.” International Journal of Social Quality 10 (1): 57-86. doi:10.3167/IJSQ.2020.100104.

WHO (World Health Organization). 2021. "WHO Coronavirus (COVID-19) Dashboard." https:// covid19.who.int/ (accessed 19 September 2021).

Xinhua News Agency. 2020a. "Xi Focus: Xi Stresses Strong Public Health System to Safeguard People's Health.” Xinhua News Agency, 2 June. http://www.news.cn/english/2020-06/ 02/c_139108964.htm.

Xinhua News Agency. 2020b. "Xi Jinping Chaired a Meeting of the Standing Committee of the Political Bureau of the CPC Central Committee to Study the Prevention and Control of the Novel Coronavirus Outbreak.” Xinhua News Agency, 25 January. http://www.gov.cn/xinwen/ 2020-01/25/content_5472188.htm.

Yang, B., L. Xiao, and K. Chen. 2020. "Ladder of Cooperation: A Study on the Joint Action of Social Organizations to Fight COVID-19." [In Chinese.] China Nonprofit Review 26 (2): 14-33.

Yao, W. 2020. "Further Leveraging the Advantages of Social Work and Voluntary Service to Promote Grassroots Social Governance: Inspiration from the Participation of Social Work and Voluntary Service Forces in COVID-19 Prevention and Control in Hubei Province." [In Chinese.] Social Work in China 12: 8-9.

Zhao, D., Y. Zhao, H. Bowen, and M. Gao. 2020. "Safety, Trust and Satisfaction: A Survey Report on Social Mentality during the Epidemic in Heilongjiang Province." In Annual Report on the Social Mentality of China, ed. Wang Junxiu, 157-181. Beijing: Social Sciences Academic Press.

Zhao, H. 2011. "Multidimensional Interpretation of 'Social Quality' and Policy Enlightenment." [In Chinese.] Jianghuai Tribune 1: 119-124.

Zhong, K. 2020. "Changes and Constants in the National Emergency Command System: A Comparative Study Based on the Cases of SARS, Influenza A and COVID-19.” [In Chinese.] Administrative Law Studies 3: 11-23.

Zhong, K., and W. Lin. 2018. "Adjustment and Change in China's Disaster Relief System: A Comparative Study Based on Typical Cases." [In Chinese.] Theory and Reform 3: 25-37.

Zhou, L. 2018. “'Bureaucratic and Economic Markets' and China’s Growth Story.” [In Chinese.] Chinese Journal of Sociology 38 (2): 1-45.

Zhu, J., C. Wang, and M. Hu. 2009. Responsibility-Cooperation-Action: NGO Participation in the Wenchuan Earthquake Case Study. Beijing: Peking University Press. 